\title{
Diffusion tensor imaging for multilevel assessment of the visual pathway: possibilities for personalized outcome prediction in autoimmune disorders of the central nervous system
}

\author{
Joseph Kuchling ${ }^{1,2}$ (I) $\cdot$ Alexander U Brandt ${ }^{1} \cdot$ Friedemann Paul $^{1,2,3} \cdot$ Michael Scheel $^{1}$
}

Received: 5 April 2017 / Accepted: 7 June 2017 / Published online: 25 July 2017

(C) European Association for Predictive, Preventive and Personalised Medicine (EPMA) 2017

\begin{abstract}
The afferent visual pathway represents the most frequently affected white matter pathway in multiple sclerosis (MS) and neuromyelitis optica spectrum disorders (NMOSD). Diffusion tensor imaging (DTI) can reveal microstructural or non-overt brain tissue damage and quantify pathological processes. DTI facilitates the reconstruction of major white matter fiber tracts allowing for the assessment of structure-function and damage-dysfunction relationships. In this review, we outline DTI studies investigating the afferent visual pathway in idiopathic optic neuritis (ON), NMOSD, and MS. Since MS damage patterns are believed to depend on multiple factors, i.e., ON (anterior visual pathway damage), inflammatory lesions (posterior visual pathway damage), and global diffuse inflammatory and neurodegenerative processes, comprehensive knowledge on different contributing factors using DTI in vivo may advance our understanding of MS disease
\end{abstract}

Michael Scheel

michael.scheel@charite.de

Joseph Kuchling

joseph.kuchling@charite.de

Alexander U Brandt

alexander.brandt@charite.de

Friedemann Paul

friedemann.paul@charite.de

1 Charité - Universitätsmedizin Berlin, corporate member of Freie Universität Berlin, Humboldt-Universität zu Berlin, and Berlin Institute of Health, Neurocure Cluster of Excellence, NeuroCure Clinical Research Center, Charitéplatz 1, D-10117 Berlin, Germany

2 Department of Neurology, Charité - Universitätsmedizin Berlin, Charitéplatz 1, D-10117 Berlin, Germany

3 Experimental and Clinical Research Center, Max Delbrueck Center for Molecular Medicine and Charité - Universitätsmedizin Berlin, Berlin, Germany pathology. Combination of DTI measures and visual outcome parameters yields the potential to improve routine clinical diagnostic procedures and may further the accuracy of individual prognosis with regard to visual function and personalized disease outcome. However, due to the inherent limitations of DTI acquisition and post-processing techniques and the so far heterogeneous and equivocal data of previous studies, evaluation of the true potential of DTI as a possible biomarker for afferent visual pathway dysfunction is still substantially limited. Further research efforts with larger longitudinal studies and standardized DTI acquisition and post-processing validation criteria are needed to overcome current DTI limitations. DTI evaluation at different levels of the visual pathway has the potential to provide markers for individual damage evaluation in the future. As an imaging biomarker, DTI may support individual outcome prediction during personalized treatment algorithms in MS and other neuroinflammatory diseases, hereby leveraging the concept of predictive, preventive, and personalized medicine in the field of clinical neuroimmunology.

Keywords Diffusion tensor imaging · DTI · Visual pathway · Multiple sclerosis · Neuromyelitis optica spectrum disorders · Optic neuritis · Predictive preventive personalized medicine

\begin{tabular}{ll}
\multicolumn{2}{l}{ Abbreviations } \\
AD & axial diffusivity \\
ADC & apparent diffusion coefficient \\
AQP4-ab & aquaporin-4-antibody \\
BOLD & blood-oxygen-level dependent \\
CNS & central nervous system \\
DTI & diffusion tensor imaging \\
DWI & diffusion-weighted imaging \\
EDSS & expanded disability status scale \\
FA & fractional anisotropy
\end{tabular}




$\begin{array}{ll}\text { FLAIR } & \text { fluid-attenuated inversion recovery } \\ \text { fMRI } & \text { functional magnetic resonance imaging } \\ \text { GCIPL } & \text { ganglion cell-inner plexiform layer } \\ \text { Gd } & \text { Gadolinium } \\ \text { HCVA } & \text { high-contrast visual acuity } \\ \text { LCVA } & \text { low-contrast visual acuity } \\ \text { LETM } & \text { longitudinally extensive transverse myelitis } \\ \text { LGN } & \text { lateral geniculate nucleus } \\ \text { MD } & \text { mean diffusivity } \\ \text { mfERG } & \text { multifocal electroretinography } \\ \text { mfVEP } & \text { multifocal visual evoked potentials } \\ \text { MOG } & \text { myelin oligodendrocyte glycoprotein } \\ \text { MRI } & \text { magnetic resonance imaging } \\ \text { MRS } & \text { magnetic resonance spectroscopy } \\ \text { MS } & \text { multiple sclerosis } \\ \text { MTR } & \text { magnetization transfer ratio } \\ \text { MV } & \text { macular volume } \\ \text { NAWM } & \text { normal-appearing white matter } \\ \text { NMOSD } & \text { neuromyelitis optica spectrum disorder } \\ \text { OCT } & \text { optical coherence tomography } \\ \text { ON } & \text { optic neuritis } \\ \text { OR } & \text { optic radiation } \\ \text { PPPM } & \text { predictive, preventive, and personalized medicine } \\ \text { RD } & \text { radial diffusivity } \\ \text { RNFL } & \text { retinal nerve fiber layer } \\ \text { ROI } & \text { region of interest } \\ \text { TBSS } & \text { tract-based spatial statistics } \\ \text { VBA } & \text { voxel-based analysis } \\ & \end{array}$

\section{Introduction}

The afferent visual pathway, longing from retinal photoreceptor cells to visual cortex area neurons, represents the most frequently affected white matter pathway in central nervous system (CNS) inflammatory disorders. Over one third of multiple sclerosis (MS) patients experience persistent visual symptoms [1]. Optic neuritis (ON) is among most frequent symptoms in MS [1-3] and a core clinical characteristic in neuromyelitis optica spectrum disorders (NMOSD) [4, 5]. Comparative evaluation of neuroinflammatory disease patterns such as $\mathrm{ON}$ and optic radiation (OR)-associated white matter lesions in vivo has the potential to advance our understanding about structural and functional visual system degradation during the complex and highly heterogeneous MS and NMOSD disease course. Although parameters that are based on routine structural magnetic resonance imaging (MRI), such as T2 lesions and gadolinium $(\mathrm{Gd})$ - enhancing plaques, represent highly relevant biomarkers for diagnosis $[4,6]$ and therapy response in randomized controlled clinical trials $[7$, 8], they provide insufficient information on functionally relevant disease severity, especially with respect to the visual system. DTI provides new markers that are sensitive and relatively specific to non-overt brain tissue damage [9]. DTI facilitates the reconstruction and investigation of the optic radiation and allows for an in vivo anatomical assessment of damage-dysfunction relationships $[9,10]$. The multimodal use of DTI together with optical coherence tomography (OCT) and visual evoked potentials (VEP) facilitates an objective assessment of the entire afferent visual system. Hence, DTI of the visual pathway combined with OCT and VEP provides a symptom-based clinical evaluation with new promising outcome parameters in therapeutic phase II and phase III neuroimmunological disease studies, especially of remyelinating and neuroprotective agents [1]. However, there is overwhelming heterogeneity and complexity in the use of visual pathway DTI and a lack of standards for DTI acquisition [11] and post-processing [12] techniques. This has led to equivocal findings [13] impeding the evaluation of DTI as a potential biomarker [9]. This review summarizes the current literature on DTI as a possible biomarker to assess visual system degradation in MS, NMOSD, and idiopathic ON. Additionally, we aim to give an overview of current DTI limitations and attempt to provide possible solutions to form a future research agenda towards a reasonable application of DTI as part of the neuro-visual assessment battery. Our article conforms with the recommendations of the "EPMA White Paper" [14].

\section{Visual pathway in neuroinflammatory diseases}

\section{Structure-function relationships in the visual pathway system}

The anterior part of the visual pathway (Fig. 1) consists of the first three neurons including retinal photo receptor cells, bipolar cells, as well as the retinal ganglion cells which convey visual information from the inner membrane of the retina via

Fig. 1 Selection of visual pathway anatomical structures and assessment methods. Important anatomical structures are displayed: retina, optic nerve, optic chiasm, and optic tract are parts of the anterior visual pathway and lateral geniculate nucleus (LGN), optic radiation, and primary visual cortex are parts of the posterior visual pathway. Optic neuritis within the optic nerve, OR lesions within the optic radiation, and V1 atrophy of the visual cortex are displayed as typical damage patterns in neuroinflammatory diseases, e.g., multiple sclerosis. Whereas HCVA and LCVA may assess the overall functionality of the visual system, other methods provide information on different visual system parts, i.e., OCT of the retina, DTI-based tractography of the optic radiation and functional MRI of the visual cortex. MfVEP evaluates latency delays along the entire pathway from optic nerve to V1 area. This figure was made by use of InkScape (https://inkscape.org/en/). OR lesion optic radiation lesion, V1 atrophy primary visual cortex atrophy, DTI diffusion tensor imaging, $O C T$ optical coherence tomography, $m f V E P$ multifocal visual evoked potentials, HCVA high-contrast visual acuity, $L C V A$ low-contrast visual acuity, $L G N$ lateral geniculate nucleus 


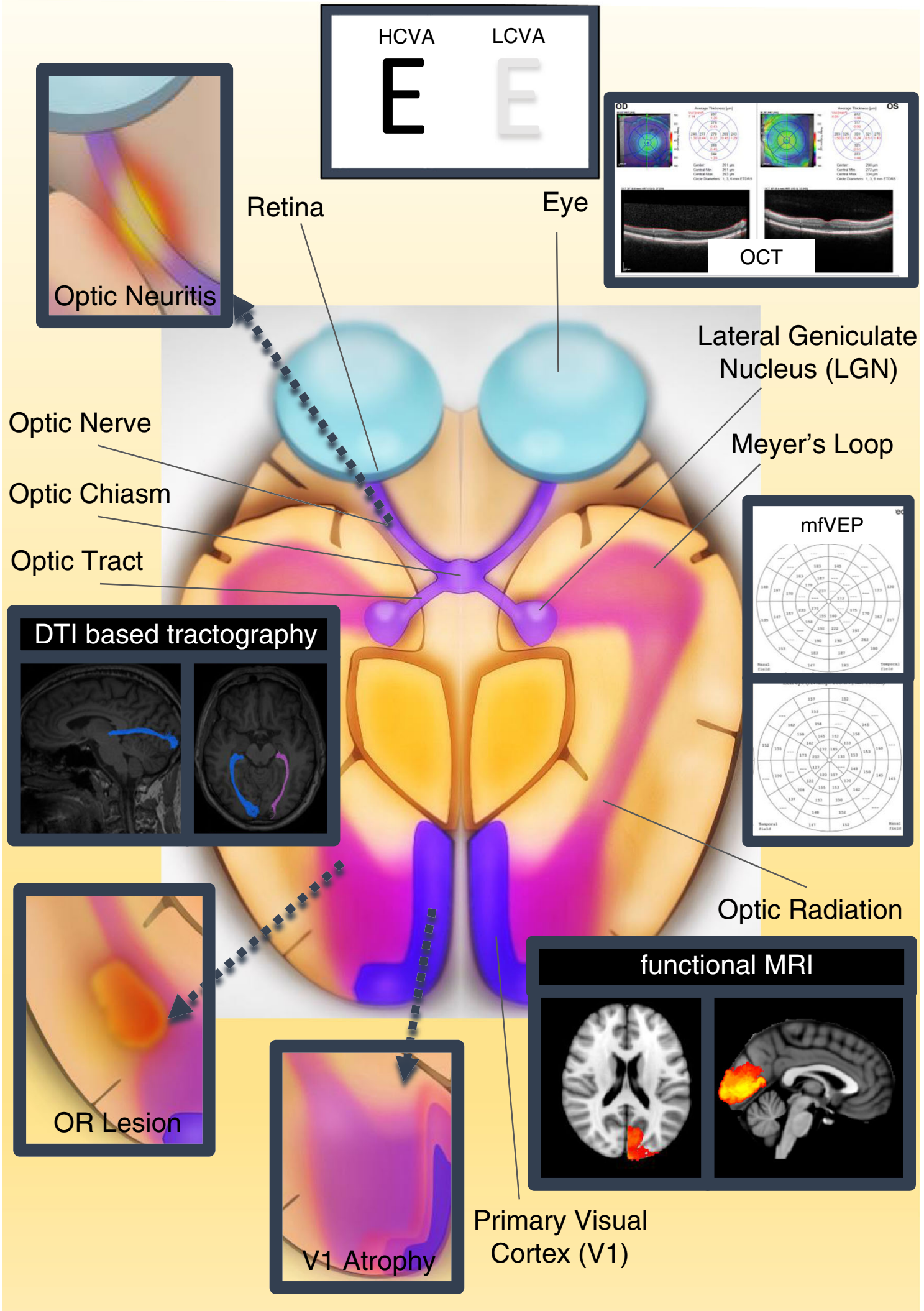

the optic nerve, the chiasm, and the optic tracts to the thalamic lateral geniculate nucleus (LGN) [15]. By forming a synapse with the fourth order neuron in the LGN, visual information is transferred to the posterior visual pathway, passing along the 
optic radiation $(\mathrm{OR})$, a white matter pathway containing strictly aligned axonal fibers with inferior visual field information conveyed in the anterior Meyer loop, to finally hit the primary visual cortex [15]. Whereas information on motion perception is transferred via the dorsal stream to parietal associative cortical areas, the ventral stream sends object-relevant information to the temporal cortex.

\section{Optic neuritis - a model of anterior visual pathway damage}

ON involves primary inflammation, demyelination, and axonal injury within the optic nerve and represents the onset symptom of MS in 20-30\% and occurs in nearly $70 \%$ of MS patients during the course of the disease [1]. Typically, MS-associated ON presents with subacute blurry vision with reduced contrast sensitivity or complete visual loss in one or - much less frequentlyboth eyes developing during hours or days associated with dyschromatopsia and pain during eye movement [16]. By contrast, NMOSD-associated ON, which is also one of the NMOSD core clinical characteristics, often presents with atypical symptoms, such as binocular affection and more pronounced visual loss with a worse recovery rate compared to MS [17-21]. Due to pathophysiological similarities between MS-associated ON and MS brain lesions with regard to inflammatory T-cell microglial activation and subsequent axonal degradation, optic neuritis serves as a concrete model of neuroinflammatory damage in the visual system [16].

\section{MS visual pathway white matter damage patterns}

Wallerian degeneration (anterograde axonal degeneration) and retrograde axonal degeneration ("dying-back" degeneration) have been suggested to occur as mid- to long-term damage after acute inflammatory changes [22], e.g., due to $\mathrm{ON}$ or after lesional development within the OR. Beyond focal axonal damage and its direct mid-term consequences, the question has emerged as to whether trans-synaptic degeneration may play an additional significant role in chronic disease development and neurodegeneration [23]. Subclinically silent tissue destruction following inflammatory damage due to $\mathrm{ON}$ or white matter lesions may serve as an example for white matter destruction in MS in general. Additionally, chronic diffuse neurodegeneration including the cortical and deep gray matter and the retina or a global diffuse inflammatory activity have been suggested to be present in long-term progressive MS course [24], but are also detectable from earliest disease stages [25-28]. This diffuse neurodegeneration may add to the degradation of visual system structures and functions and may contribute to the global and focal disease burden in MS. Therefore, a quantitative and precise assessment of microstructural damage in the visual pathway would enhance our understanding of disease pathology in MS.
NMOSD visual pathway white matter damage patterns

NMOSD is an immune-mediated inflammatory and demyelinating disease that preferentially affects the optic nerves and the spinal cord $[29,30]$ and has long believed to be a rare variant of MS. The discovery and investigation of aquaporin-4 (AQP4)-antibodies [21, 31-35] have revolutionized NMOSD diagnostics by setting the disease apart from MS [36, 37] and substantially changing NMOSD treatment [38] which considerably differs from classic diseasemodifying treatment in MS [39-48]. However, especially against the background of emerging evidence on myelin oligodendrocyte glycoprotein (MOG) - antibody-positive cases related to NMOSD, the heterogeneous pathophysiology of NMOSD still needs to be fully elucidated [49-58].

\section{Multimodal objective CNS visual pathway assessment}

Newly developed biomarkers can provide information for stratification and prediction in the setting of predictive, preventive, and personalized medicine in neurology [59]. While the entire visual pathway is profoundly susceptible to inflammatory damage [24], and although visual dysfunction has a major impact on patients' quality of life $[19,60,61]$, individual visual impairment remains highly variable and subjective among patients. Objective quantification of visual pathway damage can be approached by different interdisciplinary methods, i.e., low-contrast visual acuity (LCVA), optical coherence tomography (OCT), visual evoked potentials (VEP), electroretinography (ERG), and several advanced MRI techniques, i.e., magnetic resonance spectroscopy (MRS), magnetization transfer ratio (MTR), functional (f)MRI, and ultrahigh field imaging (Table 1) [22, 24, 62, 63].

\section{Diffusion tensor imaging}

\section{DTI indices and their correlations to CNS pathomechanisms}

DTI measures the integrity and architecture of normalappearing CNS structures by estimating displacement of water molecules along and perpendicular to white matter tracts $[13$, 73]. In each imaging voxel, DTI estimates a local diffusion profile as an ellipsoid (Fig. 2). For subsequent interpretation, the complex tensor information needs to be summarized. Finally, DTI-based indices are generated. Different DTI indices have been demonstrated to correlate with different microstructural tissue properties or pathological tissue changes (Table 2).

The most widely used parameter is the fractional anisotropy (FA) index (Fig. 3) which represents anisotropic diffusion of water molecules and is suggested to correlate with 
Table 1 Synopsis of visual pathway assessment methods and outcome parameters

\begin{tabular}{|c|c|c|c|}
\hline & $\begin{array}{l}\text { Functional vs. } \\
\text { structural }\end{array}$ & Information & Findings and correlations \\
\hline Low-contrast visual acuity (LCVA) & Functional (global) & $\begin{array}{l}\text { Sensitive to functional global visual } \\
\text { impairment and "real world" visual } \\
\text { impairment }\end{array}$ & $\begin{array}{l}\text { - LCVA correlates with visual impairment of } \\
\text { reading, facial recognition and driving } \\
\text { [64] } \\
\text { - LCVA is associated with vision-related } \\
\text { quality of life [65] }\end{array}$ \\
\hline Vision-related quality of life & Functional (global) & $\begin{array}{l}\text { Global functional assessment of patients' } \\
\text { disability related to vision }\end{array}$ & $\begin{array}{l}\text { - Vision-related quality of life correlates } \\
\text { with reduced LCVA }[19,61,65] \text { and } \\
\text { OCT retinal damage [19] }\end{array}$ \\
\hline $\begin{array}{l}\text { Optical coherence tomography } \\
\quad(\mathrm{OCT})\end{array}$ & $\begin{array}{l}\text { Structural (retina - an- } \\
\text { terior visual path- } \\
\text { way) }\end{array}$ & $\begin{array}{l}\text { Identification of structural changes } \\
\text { within the retinal tissue }\end{array}$ & $\begin{array}{l}\text { - RNFL thinning correlates with LCVA [66] } \\
\text { - RNFL thinning correlates with brain } \\
\text { atrophy [67] } \\
\text { - RNFL correlates with } 7 \mathrm{~T} \text { optic radiation } \\
\text { thinning [22] }\end{array}$ \\
\hline $\begin{array}{l}\text { Pattern visual evoked potentials } \\
\text { (VEP) and multifocal visual } \\
\text { evoked potentials (mfVEP) }\end{array}$ & $\begin{array}{l}\text { Functional (anterior } \\
\text { and posterior visual } \\
\text { pathway) }\end{array}$ & $\begin{array}{l}\text { Detection of demyelination and loss of } \\
\text { axons resulting in latency delay and/or } \\
\text { conduction block }\end{array}$ & $\begin{array}{l}\text { - Early VEP prolongation predicts } \\
\text { subsequent retinal axonal loss [68] } \\
\text { - VEP over time correlates with visual } \\
\text { cortex fMRI [69] } \\
\text { - VEP latency correlates with optic nerve } \\
\text { MTR [70] }\end{array}$ \\
\hline $\begin{array}{l}\text { Pattern electroretinography (ERG) } \\
\text { and multifocal electroretinography } \\
\text { (mfERG) }\end{array}$ & $\begin{array}{l}\text { Functional } \\
\text { (retina-anterior } \\
\text { visual pathway) }\end{array}$ & $\begin{array}{l}\text { Measurement of electrical responses of } \\
\text { retinal cell types }\end{array}$ & $\begin{array}{l}\text { - mfERG correlates with LCVA and RNFL } \\
\text { [71] }\end{array}$ \\
\hline $\begin{array}{l}\text { Magnetic resonance spectroscopy } \\
\text { (MRS) }\end{array}$ & $\begin{array}{l}\text { Structural (posterior } \\
\text { visual pathway and } \\
\text { visual cortex) }\end{array}$ & $\begin{array}{l}\text { Quantification of metabolites (e.g., } \\
N \text {-acetyl-aspartate) within voxels }\end{array}$ & $\begin{array}{l}\text { - MRS correlates with RNFL thinning in } \\
\text { ON [23] }\end{array}$ \\
\hline Magnetization transfer ratio (MTR) & $\begin{array}{l}\text { Structural (anterior and } \\
\text { posterior visual } \\
\text { pathway) }\end{array}$ & $\begin{array}{l}\text { Measurement of myelin content within } \\
\text { anatomical structures }\end{array}$ & $\begin{array}{l}\text { - Optic nerve MTR correlates with VEP } \\
\text { latency following optic neuritis [70] }\end{array}$ \\
\hline Functional MRI (fMRI) & $\begin{array}{l}\text { Functional (visual } \\
\text { cortex) }\end{array}$ & $\begin{array}{l}\text { Changes in cortical activation-based } \\
\text { BOLD effect }\end{array}$ & $\begin{array}{l}\text { - fMRI negatively correlates with VEP after } \\
\text { ON [69] } \\
\text { - Cortical activation correlates with visual } \\
\text { motion perception [72] }\end{array}$ \\
\hline Ultrahigh field MRI at $7 \mathrm{~T}$ & $\begin{array}{l}\text { Structural (posterior } \\
\text { visual pathway) }\end{array}$ & $\begin{array}{l}\text { Delineation of the optic radiation in } \mathrm{T} 2 * \\
\text { imaging }\end{array}$ & $\begin{array}{l}\text { - Correlations of OR thinning with OCT } \\
\text { RNFL and OR lesion load }[22,62,63]\end{array}$ \\
\hline
\end{tabular}

LCVA low-contrast visual acuity, OCT optical coherence tomography, $R N F L$ retinal nerve fiber layer, $7 T 7$ Tesla, NMOSD neuromyelitis optica spectrum disorders, $M S$ multiple sclerosis, $m f V E P$ multifocal visual evoked potentials, $f M R I$ functional MRI, $M T R$ magnetization transfer ratio, $m f E R G$ multifocal ERG, $M R S$ magnetic resonance spectroscopy, $O N$ optic neuritis, $B O L D$ blood-oxygen-level dependent, $O R$ optic radiation

microstructural integrity $[9,13]$. Mean diffusivity (MD) values correspond to the overall molecular water diffusion rate and reflect the status of tissue integrity at a rather global level $[9,13]$. More specific insights are provided by evaluation of axial diffusivity $(\mathrm{AD})$ and radial diffusivity $(\mathrm{RD})$ values. $\mathrm{AD}$ is discussed to represent potential axonal damage, and $\mathrm{RD}$ commonly increases when white matter demyelination and axonal density loss occur [9].

\section{DTI-based white matter assessment}

There are different approaches for the evaluation of DTI parameters. Regional assessment can be enabled by region-ofinterest (ROI) analysis [73]. Whole brain analyses are performed using voxel-based analysis (VBA) or tract-based spatial statistics (TBSS; Fig. 4) [74]. Moreover, tractography analysis can be used to confine evaluation to specific tracts. Several post-processing software techniques have been developed. A selection of the different approaches, the related software and the study groups is shown in Table 3. Deterministic tractography uses the principal eigenvector information in combination with FA and anatomical knowledge through seed and target ROIs [73]. However, deterministic tractography methods are impeded by the limited knowledge on the possible error of deviance caused by the tracking procedure [73]. On the other hand, probabilistic tractography incorporates the uncertainty into the tracking algorithm and produces connectivity metric at each voxel (Fig. 5) [75]. 


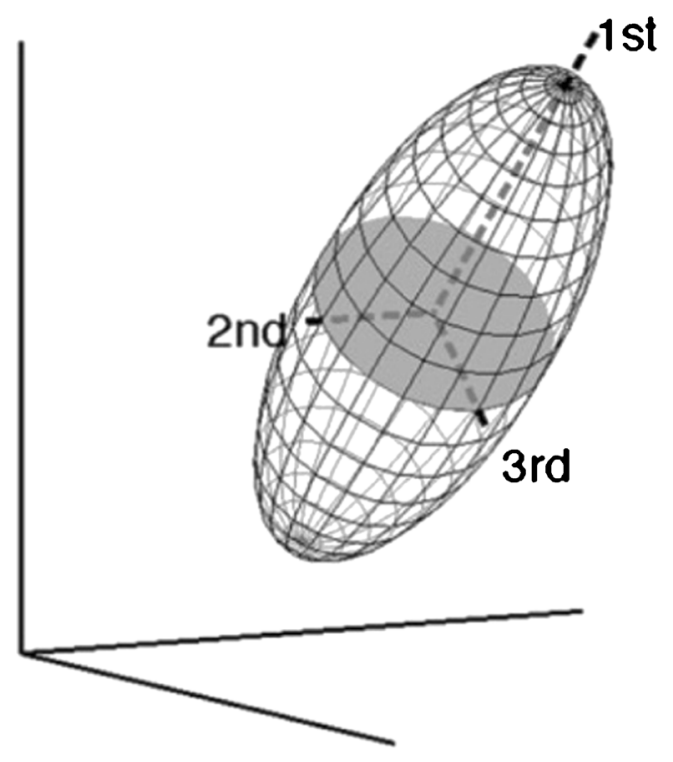

Fig. 2 Tensor ellipsoid with the main axes. Each axis has its eigenvector (orientation of the axis) and its eigenvalue (length of the axis). The 1st eigenvector is mainly used for tractography purposes; the eigenvalues are the basis for all DTI parameters (eg. fractional anisotropy)

The results of any of these evaluations can be combined with other structural and functional MRI parameters, e.g., topological T1/T2 lesion load within the optic radiation and blood-oxygen-level dependent (BOLD)-effect analyses within visual system structures. These approaches have the potential of a more specific evaluation of damage-dysfunction relationships on the anatomical and microstructural basis.

\section{Visual pathway DTI in idiopathic ON}

Few DTI studies focusing on idiopathic ON have investigated the effect of isolated ON damage on the optic nerve and on neighboring visual pathway structures. Optic nerve DTI investigations revealed increased $R D$ and decreased FA values at acute $\mathrm{ON}$ onset within the optic nerves of 33 clinically isolated ON patients compared to healthy controls [95]. No FA differences were seen within the OR of patients that were investigated less than 1 year after ON compared with healthy controls. However, patients that were investigated more than 1 year after acute $\mathrm{ON}$ exhibited significant FA decrease and $\mathrm{RD}$ increase within the OR, suggesting temporal dynamics to be a key factor in the detection of trans-synaptic neurodegeneration after $\mathrm{ON}$ with detectable OR changes after at least 1 year of acute $\mathrm{ON}$ onset. These findings were corroborated by a longitudinal study that reported a decrease in FA and an increase in $\mathrm{RD}$ within the $\mathrm{OR}$ of patients over 1 year following $\mathrm{ON}$, whereas no significant longitudinal change occurred in healthy controls [96]. Another longitudinal study that investigated 38 acute clinically isolated ON patients at presentation and 6 and 12 months after acute ON consistently found ORspecific FA reduction at an average rate of $-2.6 \%$ per annum [97]. In addition, decrease in FA as well as increase in $\mathrm{RD}$ and MD correlated with primary visual cortex thinning over 12 months. AD showed positive correlations with RNFL change in patients with no OR-specific T2 lesions [97]. In sum, ON-driven trans-synaptic degeneration can be quantified by longitudinal OR DTI and may occur as a prolonged response after isolated ON. However, heterogeneous cohorts in different studies strongly limit comparability between these studies. In one of these studies, 19 out of 38 patients initially classified as clinically isolated ON were subsequently diagnosed with MS after 12 months [97] while in the other, no status of RRMS diagnosis after 1 year was reported [96].

\section{Visual pathway DTI in NMOSD}

\section{Optic nerve DTI in NMOSD}

A DTI study investigating the optic nerve revealed reduced FA values in affected optic nerves of 28 NMOSD patients (all with an aquaporin-4-antibody [AQP4-ab] positive status) when compared to healthy control subjects [98]. However,

Table 2 Overview of DTI indices

\begin{tabular}{|c|c|c|c|c|}
\hline Parameter & Abbreviation & Unit & Formula & $\begin{array}{l}\text { Proposed pathologic } \\
\text { correlate }\end{array}$ \\
\hline Fractional anisotropy & FA & scalar & $\sqrt{\frac{1}{2}} \frac{\sqrt{\left(\lambda_{1}-\lambda_{2}\right)^{2}+\left(\lambda_{2}-\lambda_{3}\right)^{2}+\left(\lambda_{3}-\lambda_{1}\right)^{2}}}{\sqrt{\left.\lambda_{1}^{2}+\lambda_{2}^{2}+\lambda_{3}^{2}\right)}}$ & $\begin{array}{l}\text { Microstructural } \\
\text { disintegration }\end{array}$ \\
\hline Median diffusivity & MD & $\mathrm{mm}^{2} / \mathrm{s}$ & $\frac{\left(\lambda_{1}+\lambda_{2}+\lambda_{3}\right)}{3}$ & $\begin{array}{l}\text { Microstructural } \\
\text { disintegration }\end{array}$ \\
\hline Radial diffusivity & $\mathrm{RD}$ & $\mathrm{mm}^{2} / \mathrm{s}$ & $\frac{\left(\lambda_{2}+\lambda_{3}\right)}{2}$ & Myelin loss \\
\hline Axial diffusivity & $\mathrm{AD}$ & $\mathrm{mm}^{2} / \mathrm{s}$ & $\lambda_{1}$ & Axonal degradation \\
\hline
\end{tabular}

$\lambda=$ eigenvalues of the three principal tensor axes 

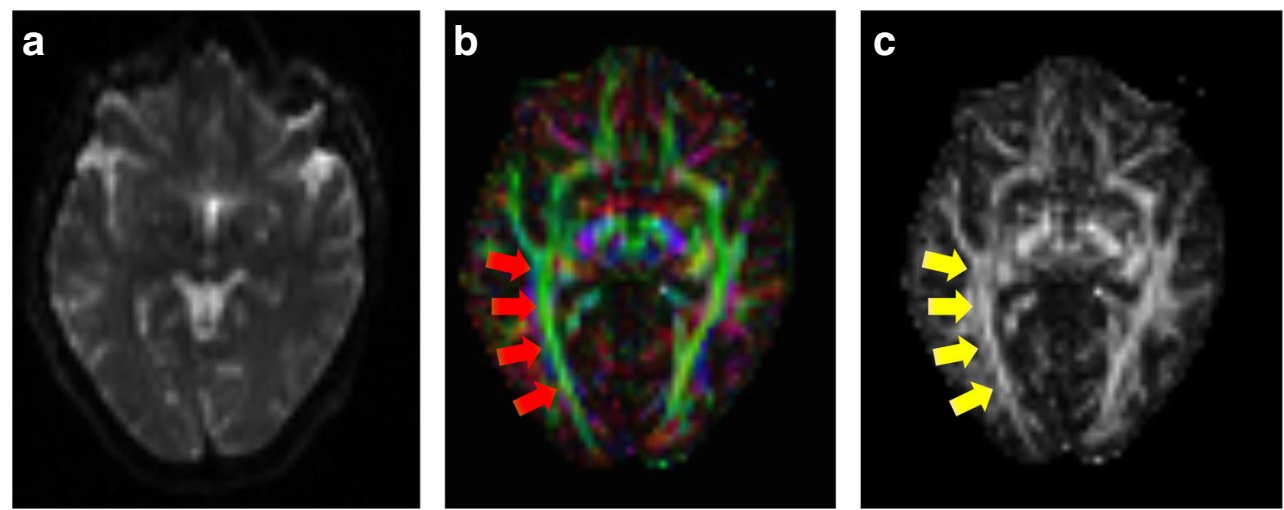

Fig. 3 Diffusion-weighted image, eigenvector map, and fractional anisotropy map. a Healthy control diffusion-weighted image used as raw image for further DTI post-processing. b Eigenvector map with encoding of eigenvector directions by RGB code (red = left-right orientation; green $=$ anterior-posterior orientation; blue $=$ inferior-superior

whether optic nerve DTI values really could serve as potential biomarkers to monitor disease progress and predict visual outcome in NMOSD patients after ON is still a matter of debate and requires more extensive efforts concerning longitudinal visual pathway DTI studies in NMOSD.

\section{Optic radiation DTI in NMOSD}

Recent investigations of DTI values within the OR showed pathological alterations of NMOSD patients compared to normal controls: first, OR MD values were consistently increased in NMOSD [76-78] suggesting microstructural integrity loss of OR fibers in NMOSD patients. Second, a significant reduction of FA within NMOSD OR was commonly observed [76, 79-81, 84, 99, 100]. Hence, microstructural damage within the optic radiation is likely to occur during the course of the disease. Third, RD increase [80] has been sporadically reported to be present in the visual system of NMOSD patients indicating demyelination processes to occur within the OR. Up to now, there were no consistent reports on $\mathrm{AD}$ changes as a correlate of potential axonal damage within the OR throughout the literature $[80,81]$.

Collectively, these findings are highly indicative of transsynaptic anterograde degeneration spreading from the optic nerves to the OR in NMOSD. One study comprising 21 NMOSD patients (90\% AQP4-ab positive) using TBSS reported parenchymal brain damage to be predominantly located in the $\mathrm{OR}$ and found FA decrease within the OR to be associated with RNFL thinning (Fig. 4) [84]. Another group consistently showed that DTI OR FA reductions were exclusively confined to visual pathway structures in NMOSD [81]. This notion is fostered by other study results reporting evidence on OR DTI value alterations only in regions of corticospinal or visual pathways [76, $100,101]$. However, there is still conflicting evidence as to whether covert tissue damage can occur independent of attackassociated lesions in NMOSD [102]. One study used DTI-based orientation) with red arrows showing highly aligned fibers of the optic radiation and $\mathbf{c}$ fractional anisotropy (FA) map with high values (white) in regions of highly aligned fiber tracts and low values (dark gray) in areas of diffuse fiber orientation (yellow arrows showing highly aligned fibers of the optic radiation corresponding to red arrows in $\mathbf{b}$ )

probabilistic OR tractography and revealed FA reductions within the OR of 25 AQP4-ab seropositive NMOSD patients [94]. Notably, OR FA was not only reduced in NMOSD patients with previous ON but FA reductions were also detectable in six NMOSD patients with longitudinally extensive transverse myelitis (LETM) without evidence of prior ON. These findings suggest microstructural changes in the afferent visual system independent of ON attack-related mechanisms, possibly due to AQP4-ab associated diffuse astrocytopathy [94]. Another article reported diffuse FA reduction within subcortical white matter structures in 21 NMOSD patients (90\% AQP4-ab positive) involving frontal, parietal, temporal, occipital, and limbic lobes [82]. Moreover, according to recent publications, widespread FA reductions were reported to be present along all brain tracts $[80,103]$. Larger longitudinal multimodal DTI studies are required to tackle the question as to whether NMOSD white matter damage is exclusively confined to attack-related anterior visual pathway and corticospinal tract degradation or extends to other CNS pathways.

\section{DTI-based differential diagnosis between NMOSD and MS}

Few studies have investigated the question as to whether DTI may help to distinguish between MS and NMOSD using multimodal MRI approaches. One study used two-dimensional histograms of apparent diffusion coefficient (ADC) and FA of the whole brain. The correct recognition rate of NMO and relapsing-remitting MS using this whole-brain DTI classification feature was $85.7 \%$ [101]. Another multimodal approach used 18 parameters from neuroimaging (among others DTI), clinical, and cognitive measures to feed a classification algorithm that enabled the distinction between MS, NMO, and healthy controls with an accuracy of $84 \%$ [104]. Specific analyses suggested more pronounced FA decrease within the optic radiations of MS patients compared to less optic radiation FA 
decrease in NMOSD. Corpus callosum ROI-based DTI investigations showed FA decrease to be more pronounced in the corpus callosum of MS patients compared to NMO with sensitivity and specificity of 92.3 and $93.5 \%$ for differentiation between both patient groups [105]. Up to now, the overall differential diagnostic capacity of DTI within the visual pathway remains questionable. To further elucidate differences and similarities in NMO and MS within the visual pathway, comparative studies including both patient groups with accurate assessment of visual pathway anatomical structures are required to dissect distinctive white matter damage patterns.
Visual pathway DTI in multiple sclerosis

DTI in MS anterior visual pathway due to ON

Optic nerve DTI in MS-ON

In general, optic nerve DTI indices (FA, MD, RD, AD) were demonstrated to be significantly different in MS patients compared to healthy controls indicating microstructural optic nerve damage in terms of demyelination and axonal degradation subsequent to optic neuritis in MS patients [106-108]. In

\section{TBSS FA SKELETON}

a
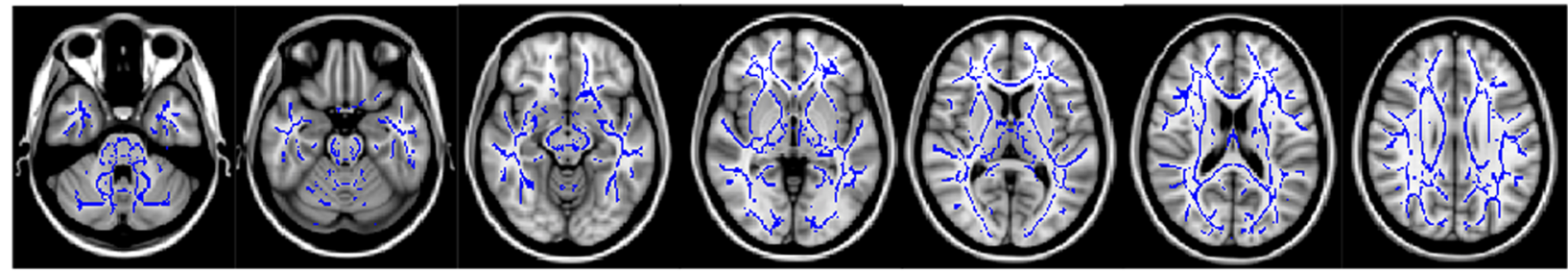

FA

Uncorrected for multiple comparison
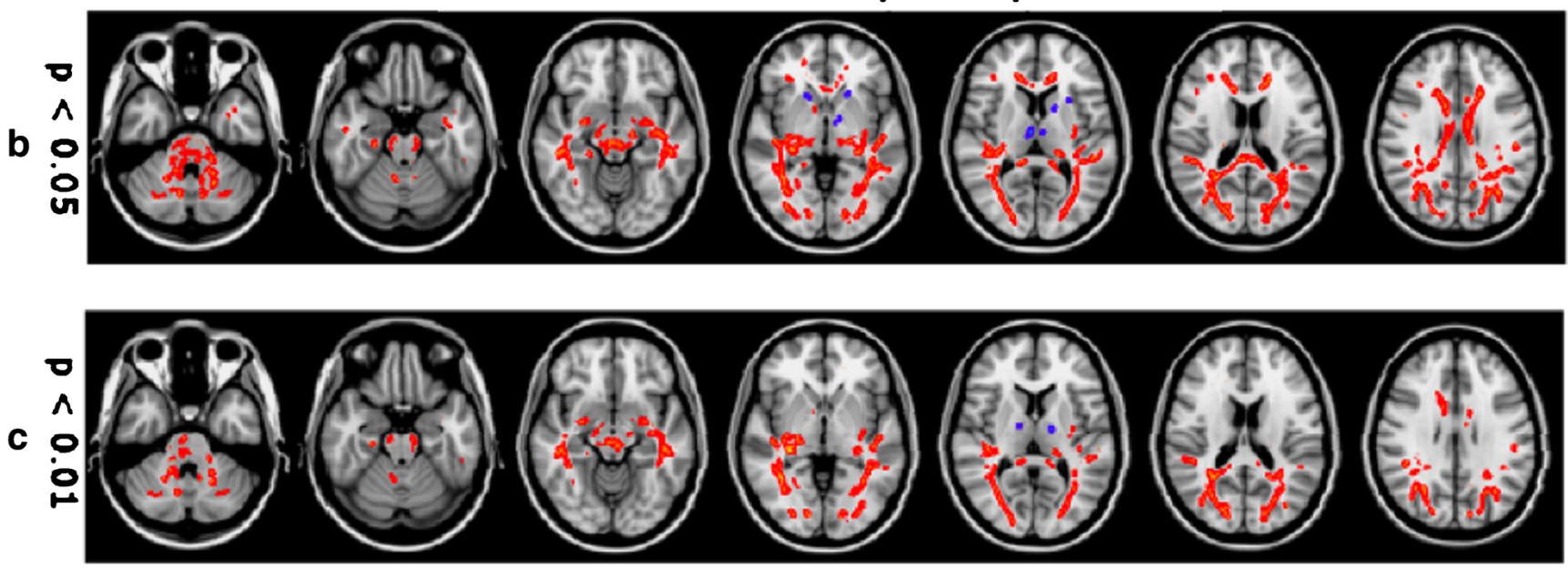

Corrected for multiple comparison

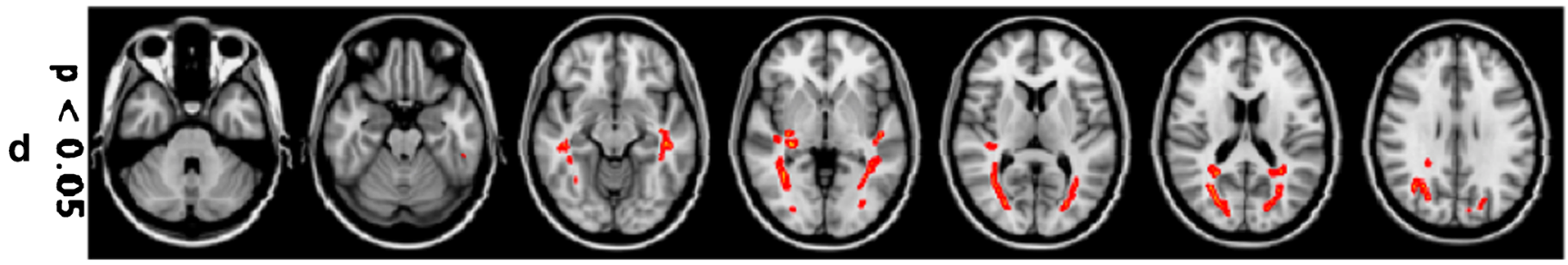

Fig. 4 TBSS analysis of patients' fiber tracts in patients with NMOSD compared to healthy controls. a TBSS mean FA skeleton of patients and healthy controls. b-d FA differences between NMOSD patients and healthy controls at $p$ values uncorrected for multiple comparisons $p<0.05$ (b) and $p<0.01$ (c), and at a $p<0.05$ fwe corrected for multiple comparisons (d). Red-yellow = reduction in NMOSD patients/ blue-light blue $=$ increase in NMOSD patients. For visualization purposes, significant skeleton tracts are thickened (tbss_fill script of fsl). b-d Are images reproduced from Pache F, Zimmermann H, Finke C, et al. (2016) Brain parenchymal damage in neuromyelitis optica spectrum disorder-a multimodal MRI study. Eur Radiol 26: 4413-4422. [84] 


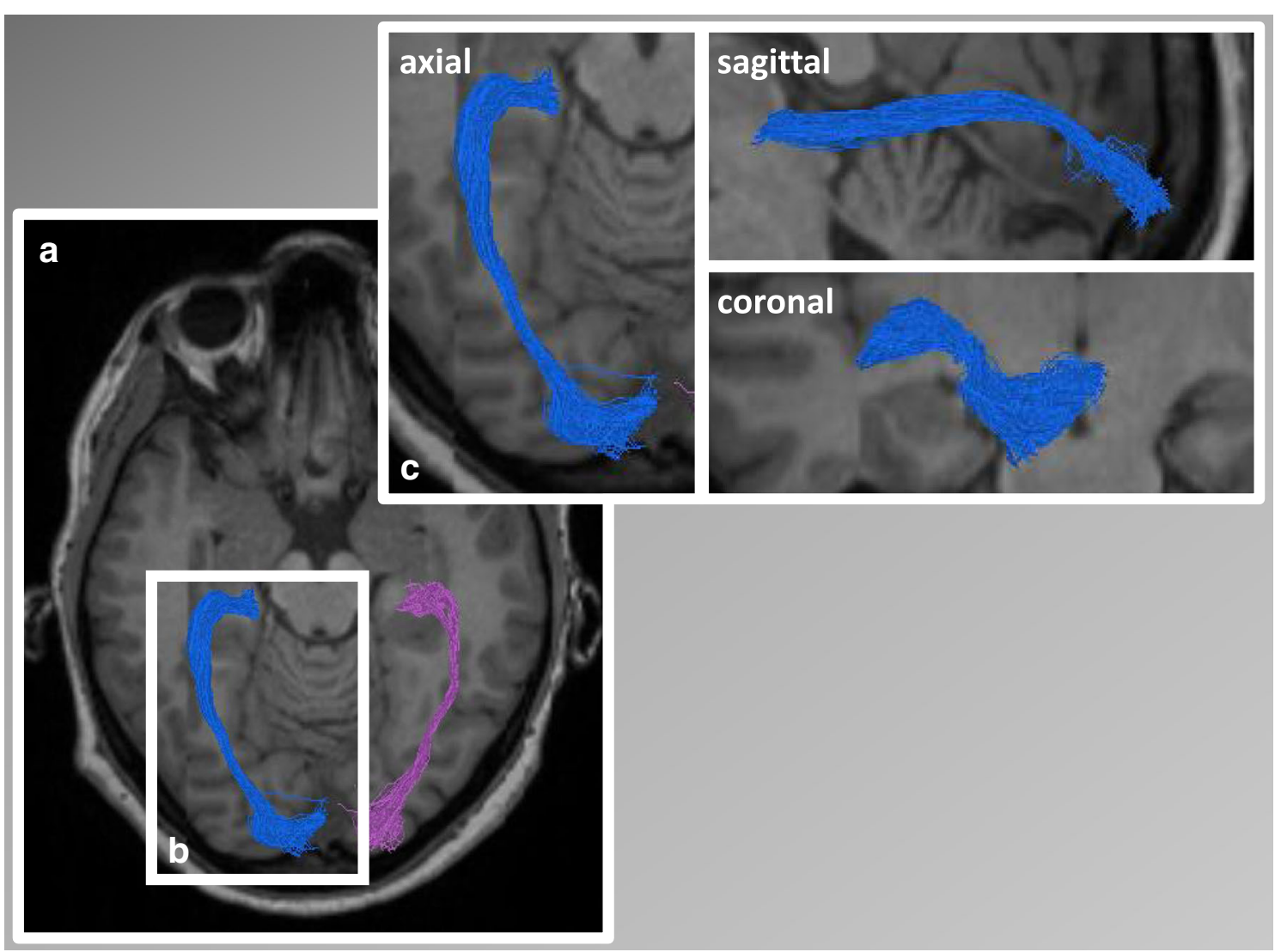

Fig. 5 Probabilistic tractography of the optic radiation. a Optic radiation fibers are calculated using probabilistic tractography (constrained spherical deconvolution [122] using MRtrix 0.2.12; Brain Research Institute, Melbourne, Australia) in a patient with neuromyelitis optica spectrum disorder (NMOSD). Optic radiation in $\mathbf{b}$ is visualized in axial, sagittal, and coronal plane (c) using DTI Quench (Vistalab Software, Stanford University)

between RNFL loss and MD/RD/AD increase to be most pronounced in secondary progressive MS ON patients indicating potential differences in the intensity of pathogenetic mechanisms between distinct MS phenotypes [109].

Although there is evidence of more pronounced DTI alterations within ON-affected optic nerves compared to DTI value changes in MS fellow eyes without prior ON [113], this notion has been questioned by an investigation of a large cohort (104 MS patients) showing both $\mathrm{ON}$-affected and non-ON-affected optic nerves to exhibit similar FA decrease with no differences between the two groups [109]. However, the same study showed correlations between FA decrease and high-contrast visual acuity (HCVA) impairment to be stronger in ON optic nerves compared to non-ON optic nerves. These results were challenged by another study investigating $23 \mathrm{MS}-\mathrm{ON}$ patients that revealed 2.5 and $1.25 \%$ contrast letter acuity in the unaffected eye to positively correlate with optic nerve FA and reported absence of these correlations when only ONaffected optic nerves of MS patients were considered [114]. Hence, there is still conflicting evidence regarding the impact of ON on associations between ON DTI indices and structural as well as functional visual outcome parameters. Consideration of ON status and subgroup analysis of affected 
Table 3 Selection of common DTI post-processing techniques to visualize the optic radiation implemented in multiple sclerosis (MS), neuromyelitis optica spectrum disorder (NMOSD) and idiopathic optic neuritis $(\mathrm{ON})$ visual pathway assessment studies

\begin{tabular}{llll}
\hline DTI post-processing approach & Software & Disease & Study group \\
\hline $\begin{array}{llll}\text { Region-of-interest (ROI)-based analysis on } \\
\text { whole-brain DTI images }\end{array}$ & SPM & NMOSD & Yu et al. [76] \\
Tract-based spatial statistics (TBSS) & DtiStudio & NMOSD & Zhao et al. [77] \\
& FSL TBSS & NMOSD & Liu et al. [78] \\
& FSL TBSS & NMOSD & Doring et al. [79] and \\
& & & Rueda-Lopes et al. [80] \\
& FSL TBSS & NMOSD & Pichiecchio et al. [81] \\
& FSL TBSS & NMOSD & von Glehn et al. [82] \\
& FSL TBSS & MS & Scheel et al. [83] \\
& FSL TBSS & NMOSD & Pache et al. [84] \\
& FSL TBSS & MS & Lobsien et al. [85] \\
& DtiStudio & MS & Dasenbrock et al. [86] \\
Deterministic tractography & DtiStudio & MS & Reich et al. [87] \\
& ConTrack & ON & Raz et al. [88, 89] \\
& ConTrack & MS & Klistorner et al. [90, 91] and \\
& & & Alsohwaeir et al. [92] \\
& FSL probtrackx & MS & Balk et al. [93] \\
& MRtrix & NMOSD & Oertel et al. [94]
\end{tabular}

SPM (Statistical Parametric Mapping - Wellcome Department of Imaging Neuroscience, University College of London). DtiStudio (Johns Hopkins University). FSL Tract-based spatial statistics (TBSS - Oxford Centre for Functional MRI of the Brain). FSL probtrackx (Oxford Centre for Functional MRI of the Brain). ConTrack (Vistalab Software, Stanford University). MRtrix package (Brain Research Institute, Melbourne, Australia)

${ }^{\mathrm{a}}$ These study groups used manual ROI delineation of the optic radiation

${ }^{\mathrm{b}}$ Dasenbrock et al. [86] used deterministic tractography to delineate the optic tract and unaffected eyes is mandatory to fully understand associations between optic nerve DTI alterations and visual function.

Several studies investigated the potential capacity for optic nerve DTI values to predict subsequent visual impairment after ON. Two studies consistently showed initial AD decrease at baseline [112] and after 1 month [111] to predict other visual outcome parameters - i.e. OCT RNFL thinning, full-field VEP latency delay - and visual acuity measures after 6 months [112] as well as OCT RNFL and multifocal VEP changes after 12 months [111]. After an initial decrease, AD values gradually increased back to normal $\mathrm{AD}$ values after a median of 18 weeks in MS ON patients. Longer AD normalization times were associated with more pronounced RNFL thinning and mfVEP amplitude loss at 12 months [111]. These findings suggest that optic nerve DTI AD values at $\mathrm{ON}$ baseline and the initial course of decreased AD values gradually increasing back to normal levels are biomarker candidates in MS patients to predict functional disability and putative recovery after acute ON. This suggests that $\mathrm{AD}$ reflects the degree of overall optic nerve axonal destruction caused by an acute inflammation. However, these findings need to be confirmed by larger optic nerve DTI studies with longer follow-up.

\section{Optic tract DTI in MS-ON}

One study [86] investigated optic tracts of $34 \mathrm{MS}$ patients without finding any correlations between DTI FA, MD, RD, or AD and ON history or between DTI parameters and EDSS. However, reduction of RNFL and total macular volume (TMV) correlated with optic tract FA decrease suggesting axonal damage in the optic nerve with retrograde or anterograde axonal degradation. In one study, reduced $\mathrm{AD}$ values in the optic tracts were associated with RNFL thinning [88], providing evidence for chronic axonal loss after acute $\mathrm{ON}$ resulting in anterograde Wallerian degeneration of the optic tracts. However, degeneration did not proceed to the optic radiations opposing the concept of anterograde trans-neuronal changes [88].

\section{Optic radiation DTI in $M S-O N$}

There is conflicting evidence with regard to the short- and long-term occurrence of trans-synaptic degeneration in MS patients after acute ON. Cross-sectional assessment (TBSS analysis) of the OR revealed correlations between the decrease of OR FA values and RNFL thinning in MS [83]. Another study showed negative correlations between mean P100 VEP and FA decline. Moreover, positive correlations between mean P100 VEP and RD were demonstrated [85], suggesting 
putative underlying anterograde trans-synaptic degeneration in the visual pathway to occur and to be quantifiable by OR DTI measurements. Whereas one study showed OR FA to be altered in ON patients compared to non-ON patients [115], another study found no changes in all OR DTI indices between the ON and non-ON group, at all [87]. Although OR DTI provides evidence on possible OR damage consecutively following after $\mathrm{ON}$, the concrete processes and mechanisms of trans-synaptic degeneration within the visual system in vivo have still not been fully elucidated. Given the heterogeneous DTI methods used in previous publications and the temporal dynamic nature of trans-synaptic degeneration lasting from acute $\mathrm{ON}$ onset until several years after attack, exact quantification by use of DTI is still hampered. Longitudinal DTI studies investigating trans-synaptic effects in narrow intervals over a period of more than 1 year subsequent to the $\mathrm{ON}$ event are highly required. Use of other advanced imaging techniques that have shown evidence of bidirectional axonal degeneration within the visual pathway, such as studies demonstrating reduced average myelin water fraction measurements within the optic radiations [116] and increased whole brain and regional visual cortex atrophy assessed by structural MRI [117] associated with OCT RNFL thinning, might be additionally useful to fully understand possible bidirectional trans-synaptic neurodegenerative processes within the visual pathway in vivo.

\section{Lesional posterior visual pathway damage in MS}

\section{Optic tract DTI after posterior visual pathway damage}

A recent study investigated optic tract DTI alterations with focus on lesional damage within the OR. Left/right asymmetry of the optic tract diameter was used as an indirect measure of optic tract primary damage. Moreover, left/right asymmetry of the OR lesion volume was measured. Analysis of optic tract asymmetry demonstrated significant associations with left/ right asymmetry of the OR lesion volume $(r=-0.42$, $p=0.001)$. This is possibly occurring due to retrograde trans-neuronal degeneration of optic tract fibers caused by OR lesions [90].

\section{Optic radiation DTI}

Correlations between OR integrity assessed by DTI indices and T2 lesion load were investigated in a study of $102 \mathrm{MS}$ patients that found FA decrease to be associated with higher T2 lesion volume [115]. Another study found higher MD values to be correlated with higher T2 lesion volume [118]. These results were consistently interpreted as a consequence of anterograde and retrograde axonal degeneration due to inflammatory lesional impact within the OR [115, 118]. One study reported regional $\mathrm{RD}$ elevation in the $\mathrm{OR}$ parts that were affected by OR-specific white matter lesions corroborating the role of $\mathrm{RD}$ as a potential marker for demyelination [91]. A significant elevation of RD in OR non-lesional fibers that correlated with increased global but not focal T2 lesion volume was found, possibly indicating OR fiber damage to be related to the overall burden of brain inflammatory activity [91]. In this study, a linear regression model was employed that, apart from lesion volume, also included disease duration, age, gender, and a history of $O N$. The model explained $38 \%$ of the observed increase in RD in non-lesional fibers and demonstrated a significant effect of $\mathrm{T} 2$ lesion volume outside of the OR and, to a lesser extent, disease duration and history of ON on RD [91]. The same investigators reported significant associations between mfVEP latency delay and increased ORspecific T2 lesion volume. They found a positive correlation between mfVEP latency delay and increase in MD, AD, and $\mathrm{RD}$ and also showed FA decrease to be associated with mfVEP latency delay [92]. A subgroup analysis in patients without a history of $\mathrm{ON}$ revealed even higher positive correlations between OR-specific T2 lesion volumes and increased VEP latencies. By contrast, this correlation was absent in fellow eyes of MS-ON patients [92]. A further study detected associations between high OR T2 lesion volume and OCT RNFL thinning as well as between increased OR T2 lesion volume and low LCVA scores [90]. These findings indicate functional impairment within the entire visual pathway caused by T2 lesions within the OR. Moreover, OR-specific T2 lesions may contribute to optic nerve and thus retinal nerve fiber layer damage due to possible retrograde trans-synaptic neurodegeneration.

\section{OR DTI as a biomarker for visual outcome}

OR DTI showed associations between FA decrease and multifocal VEP amplitude asymmetry [106] and between all four DTI indices and multifocal VEP latency prolongation [89, 92]. Whereas increased RD, MD, and AD values were positively correlated with VEP latency delay, FA showed negative correlations indicating an FA decrease to be associated with higher VEP latency delays. In a recent multiparametric visual assessment study using DTI TBSS in 222 MS patients, significant correlations between RNFL thinning and decreased FA and increased MD values of the optic radiations were reported [93]. Another article reported associations between RNFL thinning in the nasal retinal quadrant and specific FA decrease in the synaptically connected contralateral OR [87]. There is evidence that OR DTI in MS patients with no previous ON may correlate with visual acuity, whereas this correlation vanishes after the occurrence of an acute ON event [114]. However, since these results are derived from an investigation on a relatively small population of $23 \mathrm{MS}$ patients, further studies are required to clarify the capacity of OR DTI as a potential visual outcome marker in MS. In summary, combination of DTI tractography methods and T2 structural MRI 
parameters enable the detection and quantification of white matter fiber destruction within the posterior visual pathway. This multiparametric approach facilitates the distinction between (1) direct focal axonal damage due to OR lesions, (2) immediate axonal destruction of lesional fibers due to anterograde or retrograde degeneration, (3) trans-synaptic degeneration caused by prior $\mathrm{ON}$ or inflammatory lesional events, and (4) global inflammatory and diffuse neurodegenerative changes.

\section{Pitfalls and perspectives of future DTI research}

\section{DTI limitations}

To date, several limitations still hamper the validity of DTIbased research results and impede the applicability of DTI visual system assessment to routine clinical diagnostics. A multitude of distinct DTI post-processing techniques, particularly tractography methods and voxel-based approaches, have been used heterogeneously throughout all studies. This limits the comparability of findings between different research centers. Moreover, probabilistic and deterministic tractography are prone to artifacts and lack anatomical accuracy. Hence, individual tractography is still problematic and unreliable, particularly when anatomical changes due to disease pathology occur, e.g., atrophy of the ventricles or remarkable DTI FA decrease due to lesion pathology [12].

Currently, there is still no consensus on the optimal protocol parameters for DTI and DTI post-processing methods. This relates to acquisition parameters (e.g., resolution, number of directions, b-value) and also to post-processing methods (e.g., deterministic tensor-based tractography, higher-order tractography models with constrained spherical deconvolution). In light of the quantitative nature of the analysis, a major obstacle is the standardization of acquisition protocols especially across institutions. However, without standardization, the benefit of such an analysis is severely limited for clinical purposes. Only a joint effort of MRI vendors as well as international neurological and radiological societies can overcome this problem by providing guidelines and establishing the technical prerequisites for such analyses. The articles reviewed here have shown the methodological capacities of DTI and have also outlined important contributions of DTI to investigate visual pathway damage. However, the usefulness of DTI for evaluation of therapeutic approaches and prediction of visual outcomes remains to be proven, except for few studies showing evidence of optic nerve $\mathrm{AD}$ as an $\mathrm{ON}$ visual outcome predictor. Our review can therefore only provide first hints to the potential added value of DTI for describing visual pathway alterations. However, it also outlines the pitfalls and limitations of previous studies. Evaluation of normal values in DTI visual pathway studies come with several limitations. Most of the studies suffer from small sample size and lack statistical power, making inferences about larger populations or even subpopulations impossible. The majority of previous study designs was crosssectional and exploratory in nature with few hypotheses concerning statistical correlations with other visual outcome parameters and therefore did not allow for statements about longitudinal or even predictive changes of parameters and disease pathology.

\section{DTI perspectives}

The possibilities of optic nerve and optic radiation DTI and the combination of DTI methods and structural MR as well as visual outcome parameters makes DTI a useful method in current research and future clinical approaches to understand visual pathway disease pathology. This could help to foster individual tailored innovative therapeutic strategies [119].

Optic nerve AD may serve as an individual predictor of visual outcome during the acute stage of $\mathrm{ON}$ and could thus potentially become a stratification parameter to facilitate the decision between various treatment strategies (e.g., steroids, plasma exchange) at an early time point. Moreover, the distinction between axonal degradation or demyelination processes made by DTI provides the possibility to quantify positive effects of new experimental therapeutic agents including upcoming remyelinating drugs. Combination of DTI with other advanced MR parameters (i.e., atrophy measurement, MTR, MRS) may enable early classification of distinct neuroimmunological CNS diseases and identification of patients with more severe inflammation or patients with progressive axonal damage. This MR-based classification might foster the use of more tailored treatments in the sense of a "personalized neuroimmunology." Therefore, the next step will be to combine the information of DTI and other advanced MR technologies to generate patient profiles with regard to localization, quantification, functional impairment, and possible future development of visual pathway damage analogous to other biomarker profiles used in PPPM [120]. These profiles may serve as individual outcome predictors and as additive biomarkers within personalized treatment algorithms in MS and other CNS autoimmune diseases and help explain heterogeneous symptom presentations and associations, such as the association between MS and Flammer syndrome [121].

Further technical and methodological advances in the field of DTI are warranted. Standardization and validation criteria for DTI acquisition and post-processing techniques are required to improve validity, reliability, and comparability among research centers. Therefore, comparison between methods and validation by post-mortem investigations are needed. Future studies with larger sample size and concrete research questions regarding the different neuroinflammatory patterns need to be undertaken. 
Special focus will be put on longitudinal investigations to compare visual pathway degradation in NMOSD and MS by using DTI-based optic nerve and optic radiation assessment in combination with OCT and other advanced MR technologies. DTI at ultrahigh magnetic field strength at $7 \mathrm{~T}$ may yield the possibility to provide additional information compared to clinical routine MR at 1.5 or $3 \mathrm{~T}$ with regard to spatial resolution. To facilitate comparability and standardization between workgroups and to enhance power of studies, collaborative investigations are highly warranted. Preparation of supportive guidelines in the setting of PPPM might help to overcome the technological and logistical barriers concerning collaborative DTI research.

Moreover, it is imperative to consider important factors that may contribute to visual pathway damage and act as possible confounders, i.e., (1) ON, (2) OR T2 lesion load, (3) global T1/T2 lesion burden, and (4) diffuse neurodegeneration and atrophy. Eventually, future DTI studies are highly warranted to validate current findings. Further research studies will elucidate DTI's capacity to distinguish different pathological patterns and its potential as a putative biomarker in phase II and III therapy studies with the visual pathway as primary target. Future efforts must aim to combine DTI with multimodal objective visual pathway outcome parameters to facilitate individual pathology stratification and to provide personalized diagnostic measures in neuroimmunology.

\section{Compliance with ethical standards}

Conflicts of interest JK received conference registration fees from Biogen and financial research support from Krankheitsbezogenes Kompetenznetzwerk Multiple Sklerose (KKNMS), not related to this work. AUB served on the scientific advisory board for Biogen; received travel funding and/or speaker honoraria from Novartis and Biogen; has patents pending from Method and System for Optic Nerve Head Shape Quantification, perceptive visual computing based postural control analysis, multiple sclerosis biomarker, and perceptive sleep motion analysis; consulted for Nexus and Motognosis; and received research support from Novartis Pharma, Biogen Idec, BMWi, BMBF, and Guthy Jackson Charitable Foundation. Go to Neurology.org/nn for full disclosure forms. The Article Processing Charge was funded by the authors. FP serves on the scientific advisory board for Novartis; received speaker honoraria and travel funding from Bayer, Novartis, Biogen Idec, Teva, Sanofi-Aventis/ Genzyme, Merck Serono, Alexion, Chugai, MedImmune, and Shire; is an academic editor for PLoS One, is an associate editor for Neurology ${ }^{\circledR}$ Neuroimmunology \& Neuroinflammation; consulted for SanofiGenzyme, Biogen Idec, MedImmune, Shire, and Alexion; received research support from Bayer, Novartis, Biogen Idec, Teva, Sanofi-Aventis/ Genzyme, Alexion, Merck Serono, German Research Council, Werth Stiftung of the City of Cologne, German Ministry of Education and Research, Arthur Arnstein Stiftung Berlin, EU FP7 Framework Program, Arthur Arnstein Foundation Berlin, Guthy Jackson Charitable Foundation, and National Multiple Sclerosis of the USA. MS states no conflicts of interest.

Funding This study was funded by NeuroCure Cluster of Excellence (DFG Exc 257), $\mathrm{Neu}^{2}$ funding, and EXIST program.

\section{References}

1. Balcer LJ, Miller DH, Reingold SC, Cohen JA. Vision and visionrelated outcome measures in multiple sclerosis. Brain. 2015;138: $11-27$.

2. Galetta SL, Villoslada P, Levin N, Shindler K, Ishikawa H, Parr E, et al. Acute optic neuritis: unmet clinical needs and model for new therapies. Neurol. Neuroimmunol. Neuroinflamm. 2015;2:e135.

3. Petzold A, Wattjes MP, Costello F, Flores-Rivera J, Fraser CL, Fujihara K, et al. The investigation of acute optic neuritis: a review and proposed protocol. Nat Rev Neurol. 2014;10:447-58.

4. Wingerchuk DM, Banwell B, Bennett JL, Cabre P, Carroll W, Chitnis $\mathrm{T}$, et al. International consensus diagnostic criteria for neuromyelitis optica spectrum disorders. Neurology. 2016;86: 491-2.

5. Jarius S, Wildemann B, Paul F. Neuromyelitis optica: clinical features, immunopathogenesis and treatment. Clin Exp Immunol. 2014;176:149-64.

6. Polman CH, Reingold SC, Banwell B, Clanet M, Cohen JA, Filippi M, et al. Diagnostic criteria for multiple sclerosis: 2010 revisions to the McDonald criteria. Ann Neurol. 2011;69:292302.

7. Filippi M, Rocca MA, De Stefano N, Enzinger C, Fisher E, Horsfield MA, et al. Magnetic resonance techniques in multiple sclerosis: the present and the future. Arch Neurol. 2011;68:1514 20.

8. Kim HJ, Paul F, Lana-Peixoto MA, Tenembaum S, Asgari N, Palace J, et al. MRI characteristics of neuromyelitis optica spectrum disorder: an international update. Neurology. 2015;84:116573.

9. Inglese M, Bester M. Diffusion imaging in multiple sclerosis: research and clinical implications. NMR Biomed. 2010;23:86572.

10. Kremer S, Renard F, Achard S, Lana-Peixoto MA, Palace J, Asgari $\mathrm{N}$, et al. Use of advanced magnetic resonance imaging techniques in neuromyelitis optica spectrum disorder. JAMA Neurol. 2015;72:815.

11. Pagani E, Hirsch JG, Pouwels PJW, Horsfield MA, Perego E, Gass A, et al. Intercenter differences in diffusion tensor MRI acquisition. J Magn Reson Imaging. 2010;31:1458-68.

12. Thomas C, Ye FQ, Irfanoglu MO, Modi P, Saleem KS, Leopold DA, et al. Anatomical accuracy of brain connections derived from diffusion MRI tractography is inherently limited. Proc Natl Acad Sci USA. 2014;111:16574-9.

13. Assaf Y, Pasternak O. Diffusion tensor imaging (DTI)-based white matter mapping in brain research: a review. J Mol Neurosci. 2008;34:51-61.

14. Golubnitschaja O, Costigliola V, EPMA. General report \& recommendations in predictive, preventive and personalised medicine 2012: white paper of the European Association of Predictive, Preventive and Personalised Medicine. EPMA J. 2012;3:1-53.

15. Swienton DJ, Thomas AG. The visual pathway-functional anatomy and pathology. Semin Ultrasound CT MRI. 2014;35:487503.

16. Toosy AT, Mason DF, Miller DH. Optic neuritis. Lancet Neurol. 2014;13:83-99.

17. Fernandes DB, Ramos Rde I, Falcochio C, Apóstolos-Pereira S, Callegaro D, MLR M. Comparison of visual acuity and automated perimetry findings in patients with neuromyelitis optica or multiple sclerosis after single or multiple attacks of optic neuritis. J Neuroophthalmol. 2012;32:102-6.

18. Zekeridou A, Lennon VA. Aquaporin-4 autoimmunity. Neurol Neuroimmunol Neuroinflamm. 2015;2:e110.

19. Schmidt F, Zimmermann H, Mikolajczak J, Oertel FC, Pache F, Weinhold M, et al. Severe structural and functional visual system 
damage leads to profound loss of vision-related quality of life in patients with neuromyelitis optica spectrum disorders. Mult Scler Relat Disord. 2017;11:45-50.

20. Schneider E, Zimmermann H, Oberwahrenbrock T, Kaufhold F, Kadas EM, Petzold A, et al. Optical coherence tomography reveals distinct patterns of retinal damage in neuromyelitis optica and multiple sclerosis. PLoS One. 2013;8:e66151.

21. Melamed E, Levy M, Waters PJ, Sato DK, Bennett JL, John GR, et al. Update on biomarkers in neuromyelitis optica. Neurol Neuroimmunol Neuroinflamm. 2015;2:e134.

22. Sinnecker T, Oberwahrenbrock T, Metz I, Zimmermann H, Pfueller CF, Harms L, et al. Optic radiation damage in multiple sclerosis is associated with visual dysfunction and retinal thinning - an ultrahigh-field MR pilot study. Eur Radiol. 2015;25: $122-31$.

23. Gabilondo I, Martínez-Lapiscina EH, Martínez-Heras E, FragaPumar E, Llufriu S, Ortiz S, et al. Trans-synaptic axonal degeneration in the visual pathway in multiple sclerosis: axonal degeneration in MS. Ann Neurol. 2014;75:98-107.

24. Martínez-Lapiscina EH, Sanchez-Dalmau B, Fraga-Pumar E, Ortiz-Perez S, Tercero-Uribe AI, Torres-Torres R, et al. The visual pathway as a model to understand brain damage in multiple sclerosis. Mult Scler J. 2014;20:1678-85.

25. Oberwahrenbrock T, Ringelstein M, Jentschke S, Deuschle K, Klumbies K, Bellmann-Strobl J, et al. Retinal ganglion cell and inner plexiform layer thinning in clinically isolated syndrome. Mult Scler. 2013;19:1887-95.

26. Oberwahrenbrock T, Schippling S, Ringelstein M, Kaufhold F, Zimmermann H, Keser N, et al. Retinal damage in multiple sclerosis disease subtypes measured by high-resolution optical coherence tomography. Mult Scler Int. 2012;2012:530305.

27. Azevedo CJ, Overton E, Khadka S, Buckley J, Liu S, Sampat M, et al. Early CNS neurodegeneration in radiologically isolated syndrome. Neurol Neuroimmunol Neuroinflamm. 2015;2:e102.

28. Sinnecker T, Mittelstaedt P, Dörr J, Pfueller CF, Harms L, Niendorf T, et al. Multiple sclerosis lesions and irreversible brain tissue damage: a comparative ultrahigh-field strength magnetic resonance imaging study. Arch Neurol. 2012;69:739-45.

29. Pfueller CF, Paul F. Imaging the visual pathway in neuromyelitis optica. Mult Scler Int. 2011;2011:1-869814.

30. Sinnecker T, Schumacher S, Mueller K, Pache F, Dusek P, Harms $\mathrm{L}$, et al. MRI phase changes in multiple sclerosis vs neuromyelitis optica lesions at 7 T. Neurol Neuroimmunol Neuroinflamm. 2016;3:e259.

31. Metz I, Beißbarth T, Ellenberger D, Pache F, Stork L, Ringelstein $\mathrm{M}$, et al. Serum peptide reactivities may distinguish neuromyelitis optica subgroups and multiple sclerosis. Neurol Neuroimmunol Neuroinflamm. 2016;3:e204.

32. Takeshita Y, Obermeier B, Cotleur AC, Spampinato SF, Shimizu F, Yamamoto E, et al. Effects of neuromyelitis optica-IgG at the blood-brain barrier in vitro. Neurol Neuroimmunol Neuroinflamm. 2017;4:e311.

33. Majed M, Fryer JP, McKeon A, Lennon VA, Pittock SJ. Clinical utility of testing AQP4-IgG in CSF: guidance for physicians. Neurol Neuroimmunol Neuroinflamm. 2016;3:e231.

34. Kitley J, Woodhall M, Leite MI, Palace J, Vincent A, Waters P. Aquaporin-4 antibody isoform binding specificities do not explain clinical variations in NMO. Neurol Neuroimmunol Neuroinflamm. 2015;2:e121.

35. Sepúlveda M, Armangué T, Sola-Valls N, Arrambide G, MecaLallana JE, Oreja-Guevara C, et al. Neuromyelitis optica spectrum disorders: comparison according to the phenotype and serostatus. Neurol Neuroimmunol Neuroinflamm. 2016;3:e225.

36. Bennett JL, O'Connor KC, Bar-Or A, Zamvil SS, Hemmer B, Tedder TF, et al. B lymphocytes in neuromyelitis optica. Neurol Neuroimmunol Neuroinflamm. 2015;2:e104.
37. Jarius S, Paul F, Franciotta D, Waters P, Zipp F, Hohlfeld R, et al. Mechanisms of disease: aquaporin-4 antibodies in neuromyelitis optica. Nat Clin Pract Neurol. 2008;4:202-14.

38. Neuromyelitis Optica Study Group (NEMOS), Trebst C, Jarius S, Berthele A, Paul F, Schippling S, et al. Update on the diagnosis and treatment of neuromyelitis optica: recommendations of the Neuromyelitis Optica Study Group (NEMOS). J Neurol. 2014;261:1-16.

39. Valentino P, Marnetto F, Granieri L, Capobianco M, Bertolotto A. Aquaporin-4 antibody titration in NMO patients treated with rituximab: a retrospective study. Neurol Neuroimmunol Neuroinflamm. 2017;4:e317.

40. Chavarro VS, Mealy MA, Simpson A, Lacheta A, Pache F, Ruprecht $\mathrm{K}$, et al. Insufficient treatment of severe depression in neuromyelitis optica spectrum disorder. Neurol. Neuroimmunol. Neuroinflamm. 2016;3:e286.

41. Davoudi V, Keyhanian K, Bove RM, Chitnis T. Immunology of neuromyelitis optica during pregnancy. Neurol Neuroimmunol Neuroinflamm. 2016;3:e288.

42. Bar-Or A, Steinman L, Behne JM, Benitez-Ribas D, Chin PS, Clare-Salzler M, et al. Restoring immune tolerance in neuromyelitis optica: part II. Neurol Neuroimmunol Neuroinflamm. 2016;3:e277.

43. Steinman L, Bar-Or A, Behne JM, Benitez-Ribas D, Chin PS, Clare-Salzler M, et al. Restoring immune tolerance in neuromyelitis optica: part I. Neurol Neuroimmunol Neuroinflamm. 2016;3: e276.

44. Nosadini M, Alper G, Riney CJ, Benson LA, Mohammad SS, Ramanathan S, et al. Rituximab monitoring and redosing in pediatric neuromyelitis optica spectrum disorder. Neurol Neuroimmunol Neuroinflamm. 2016;3:e188.

45. Kornberg MD, Newsome SD. Unmasking and provoking severe disease activity in a patient with NMO spectrum disorder. Neurol Neuroimmunol Neuroinflamm. 2015;2:e66.

46. Kowarik MC, Hoshi M, Hemmer B, Berthele A. Failure of alemtuzumab as a rescue in a NMOSD patient treated with rituximab. Neurol Neuroimmunol Neuroinflamm. 2016;3:e208.

47. Kleiter I, Hellwig K, Berthele A, Kümpfel T, Linker RA, Hartung $\mathrm{H}-\mathrm{P}$, et al. Failure of natalizumab to prevent relapses in neuromyelitis optica. Arch Neurol. 2012;69:239-45.

48. Ayzenberg I, Schöllhammer J, Hoepner R, Hellwig K, Ringelstein $\mathrm{M}$, Aktas $\mathrm{O}$, et al. Efficacy of glatiramer acetate in neuromyelitis optica spectrum disorder: a multicenter retrospective study. J Neurol. 2016;263:575-82.

49. Zamvil SS, Slavin A. Does MOG Ig-positive AQP4-seronegative opticospinal inflammatory disease justify a diagnosis of NMO spectrum disorder? Neurol Neuroimmunol Neuroinflamm. 2015;2: 62 .

50. Jarius S, Kleiter I, Ruprecht K, Asgari N, Pitarokoili K, Borisow $\mathrm{N}$, et al. MOG-IgG in NMO and related disorders: a multicenter study of 50 patients. Part 3: Brainstem involvement - frequency, presentation and outcome. J Neuroinflammation. 2016:13-281.

51. Pache F, Zimmermann H, Mikolajczak J, Schumacher S, Lacheta A, Oertel FC, et al. MOG-IgG in NMO and related disorders: a multicenter study of 50 patients. Part 4: Afferent visual system damage after optic neuritis in MOG-IgG-seropositive versus AQP4-IgGseropositive patients. J Neuroinflammation. 2016:13-282.

52. Jarius S, Ruprecht K, Kleiter I, Borisow N, Asgari N, Pitarokoili $\mathrm{K}$, et al. MOG-IgG in NMO and related disorders: a multicenter study of 50 patients. Part 2: Epidemiology, clinical presentation, radiological and laboratory features, treatment responses, and long-term outcome. J Neuroinflammation. 2016:13-280.

53. Jarius S, Ruprecht K, Kleiter I. MOG-IgG in NMO and related disorders: a multicenter study of 50 patients. Part 1: Frequency, syndrome specificity, influence of disease activity, long-term 
course, association with AQP4-IgG, and origin. J Neuroinflammation. 2016:13-279.

54. Spadaro M, Gerdes LA, Krumbholz M, Ertl-Wagner B, Thaler FS, Schuh E, et al. Autoantibodies to MOG in a distinct subgroup of adult multiple sclerosis. Neurol Neuroimmunol Neuroinflamm. 2016;3:e257.

55. Kim S-M, Woodhall MR, Kim J-S, Kim S-J, Park KS, Vincent A, et al. Antibodies to MOG in adults with inflammatory demyelinating disease of the CNS. Neurol Neuroimmunol Neuroinflamm. 2015;2:e163.

56. Chalmoukou K, Alexopoulos H, Akrivou S, Stathopoulos P, Reindl M, Dalakas MC. Anti-MOG antibodies are frequently associated with steroid-sensitive recurrent optic neuritis. Neurol Neuroimmunol Neuroinflamm. 2015;2:e131.

57. Waters P, Woodhall M, O'Connor KC, Reindl M, Lang B, Sato DK, et al. MOG cell-based assay detects non-MS patients with inflammatory neurologic disease. Neurol Neuroimmunol Neuroinflamm. 2015;2:e89.

58. Reindl M, Rostasy K. MOG antibody-associated diseases. Neurol Neuroimmunol Neuroinflamm. 2015;2:e60.

59. Polivka J, Polivka J, Krakorova K, Peterka M, Topolcan O. Current status of biomarker research in neurology. EPMA J. 2016;7:14

60. Heesen C, Bohm J, Reich C, Kasper J, Goebel M, Gold S. Patient perception of bodily functions in multiple sclerosis: gait and visual function are the most valuable. Mult Scler. 2008;14:988-91.

61. Schinzel J, Zimmermann H, Paul F, Ruprecht K, Hahn K, Brandt A, et al. Relations of low contrast visual acuity, quality of life and multiple sclerosis functional composite: a cross-sectional analysis. BMC Neurol. 2014;14:31.

62. Sinnecker T, Kuchling J, Dusek P, Dörr J, Niendorf T, Paul F, et al. Ultrahigh field MRI in clinical neuroimmunology: a potential contribution to improved diagnostics and personalised disease management. EPMA J. 2015;6:16.

63. Kuchling J, Sinnecker T, Bozin I, Dörr J, Madai VI, Sobesky J, et al. Ultrahigh field MRI in context of neurological diseases. Nervenarzt. 2014;85:445-58.

64. Leat SJ, Legge GE, Bullimore MA. What is low vision? A reevaluation of definitions. Optom Vis Sci. 1999;76:198-211.

65. Mowry EM, Loguidice MJ, Daniels AB, Jacobs DA, Markowitz CE, Galetta SL, et al. Vision related quality of life in multiple sclerosis: correlation with new measures of low and high contrast letter acuity. J Neurol Neurosurg Psychiatry. 2009;80:767-72.

66. Bock M, Brandt AU, Kuchenbecker J, Dorr J, Pfueller CF, Weinges-Evers N, et al. Impairment of contrast visual acuity as a functional correlate of retinal nerve fibre layer thinning and total macular volume reduction in multiple sclerosis. Br J Ophthalmol. 2012;96:62-7.

67. Dörr J, Wernecke KD, Bock M, Gaede G, Wuerfel JT, Pfueller CF, et al. Association of retinal and macular damage with brain atrophy in multiple sclerosis. PLoS One. 2011;6:e18132.

68. Henderson APD, Altmann DR, Trip SA, Miszkiel KA, Schlottmann PG, Jones SJ, et al. Early factors associated with axonal loss after optic neuritis. Ann Neurol. 2011;70:955-63.

69. Benoliel T, Raz N, Ben-Hur T, Levin N. Cortical functional modifications following optic neuritis. Mult Scler. 2017;23:220-7.

70. Hickman SJ, Toosy AT, Jones SJ, Altmann DR, Miszkiel KA, MacManus DG, et al. Serial magnetization transfer imaging in acute optic neuritis. Brain. 2004;127:692-700.

71. Schnurman ZS, Frohman TC, Beh SC, Conger D, Conger A, Saidha S, et al. Retinal architecture and mfERG: optic nerve head component response characteristics in MS. Neurology. 2014;82: 1888-96.

72. Raz N, Dotan S, Benoliel T, Chokron S, Ben-Hur T, Levin N. Sustained motion perception deficit following optic neuritis: behavioral and cortical evidence. Neurology. 2011;76:2103-11.
73. Hasan KM, Walimuni IS, Abid H, Hahn KR. A review of diffusion tensor magnetic resonance imaging computational methods and software tools. Comput Biol Med. 2011;41:1062-72.

74. Smith SM, Jenkinson M, Johansen-Berg H, Rueckert D, Nichols TE, Mackay CE, et al. Tract-based spatial statistics: voxelwise analysis of multi-subject diffusion data. NeuroImage. 2006;31: 1487-505.

75. Mukherjee P, Berman JI, Chung SW, Hess CP, Henry RG. Diffusion tensor MR imaging and fiber tractography: theoretic underpinnings. Am J Neuroradiol. 2008;29:632-41.

76. Yu CS, Lin FC, Li KC, Jiang TZ, Zhu CZ, Qin W, et al. Diffusion tensor imaging in the assessment of normal-appearing brain tissue damage in relapsing neuromyelitis optica. Am J Neuroradiol. 2006;27:1009-15.

77. Zhao D-D, Zhou H-Y, Wu Q-Z, Liu J, Chen X-Y, He D, et al. Diffusion tensor imaging characterization of occult brain damage in relapsing neuromyelitis optica using 3.0 T magnetic resonance imaging techniques. NeuroImage. 2012;59:3173-7.

78. Liu Y, Duan Y, He Y, Yu C, Wang J, Huang J, et al. A tract-based diffusion study of cerebral white matter in neuromyelitis optica reveals widespread pathological alterations. Mult Scler. 2012;18: 1013-21.

79. Doring TM, Lopes FCR, Kubo TTA, Tukamoto G, Kimura MC, Strecker RM, et al. Neuromyelitis optica: a diffusional kurtosis imaging study. Am J Neuroradiol. 2014;35:2287-92.

80. Rueda Lopes FC, Doring T, Martins C, Cabral FC, Malfetano FR, Pereira VC, et al. The role of demyelination in neuromyelitis optica damage: diffusion-tensor MR imaging study. Radiology. 2012;263:235-42.

81. Pichiecchio A, Tavazzi E, Poloni G, Ponzio M, Palesi F, Pasin M, et al. Advanced magnetic resonance imaging of neuromyelitis optica: a multiparametric approach. Mult Scler. 2012;18:817-24.

82. von Glehn F, Jarius S, Lira RPC, Ferreira MCA, von Glehn FHR, Costa E, et al. Structural brain abnormalities are related to retinal nerve fiber layer thinning and disease duration in neuromyelitis optica spectrum disorders. Mult Scler. 2014;20:1189-97.

83. Scheel M, Finke C, Oberwahrenbrock T, Freing A, Pech L, Schlichting J, et al. Retinal nerve fibre layer thickness correlates with brain white matter damage in multiple sclerosis: a combined optical coherence tomography and diffusion tensor imaging study. Mult Scler. 2014;20:1904-7.

84. Pache F, Zimmermann H, Finke C, Lacheta A, Papazoglou S, Kuchling $\mathrm{J}$, et al. Brain parenchymal damage in neuromyelitis optica spectrum disorder - a multimodal MRI study. Eur Radiol. 2016;26:4413-22.

85. Lobsien D, Ettrich B, Sotiriou K, Classen J, Then Bergh F, Hoffmann K-T. Whole-brain diffusion tensor imaging in correlation to visual-evoked potentials in multiple sclerosis: a tract-based spatial statistics analysis. Am J Neuroradiol. 2014;35:2076-81.

86. Dasenbrock HH, Smith SA, Ozturk A, Farrell SK, Calabresi PA, Reich DS. Diffusion tensor imaging of the optic tracts in multiple sclerosis: association with retinal thinning and visual disability. J Neuroimaging. 2011;21:e41-9.

87. Reich DS, Smith SA, Gordon-Lipkin EM, Ozturk A, Caffo BS, Balcer LJ, et al. Damage to the optic radiation in multiple sclerosis is associated with retinal injury and visual disability. Arch Neurol. 2009;66:998-1006.

88. Raz N, Bick AS, Ben-Hur T, Levin N. Focal demyelinative damage and neighboring white matter integrity: an optic neuritis study. Mult Scler. 2015;21:562-71.

89. Raz N, Chokron S, Ben-Hur T, Levin N. Temporal reorganization to overcome monocular demyelination. Neurology. 2013;81:7029 .

90. Klistorner A, Sriram P, Vootakuru N, Wang C, Barnett MH, Garrick R, et al. Axonal loss of retinal neurons in multiple 
sclerosis associated with optic radiation lesions. Neurology. 2014;82:2165-72.

91. Klistorner A, Vootakuru N, Wang C, Yiannikas C, Graham SL, Parratt J, et al. Decoding diffusivity in multiple sclerosis: analysis of optic radiation lesional and non-lesional white matter. PLoS One. 2015;10:e122114.

92. Alshowaeir D, Yiannikas C, Garrick R, Parratt J, Barnett MH, Graham SL, et al. Latency of multifocal visual evoked potentials in nonoptic neuritis eyes of multiple sclerosis patients associated with optic radiation lesions. Invest. Opthalmol. Vis. Sci. 2014;55: 3758-64.

93. Balk LJ, Steenwijk MD, Tewarie P, Daams M, Killestein J, Wattjes MP, et al. Bidirectional trans-synaptic axonal degeneration in the visual pathway in multiple sclerosis. J Neurol Neurosurg Psychiatry. 2015;86:419-24.

94. Oertel FC, Kuchling J, Zimmermann H, Chien C, Schmidt F, Knier B, et al. Microstructural visual system changes in AQP4antibody-seropositive NMOSD. Neurol Neuroimmunol Neuroinflamm. 2017;4:e334.

95. Li M, Li J, He H, Wang Z, Lv B, Li W, et al. Directional diffusivity changes in the optic nerve and optic radiation in optic neuritis. Br J Radiol. 2011;84:304-14.

96. Tur C, Goodkin O, Altmann D. Longitudinal evidence for anterograde trans-synaptic degeneration after optic neuritis. Brain. 2016;139:816-28.

97. Kolbe S, van der Walt A, Butzkueven H. Serial diffusion tensor imaging of the optic radiations after acute optic neuritis. J Ophthalmol. 2016;2016:2764538.

98. Chen Z, Lou X, Liu M, Huang D, Wei S, Yu S, et al. Assessment of optic nerve impairment in patients with neuromyelitis optica by MR diffusion tensor imaging. PLoS One. 2015;10:e0126574.

99. Kimura MCG, Doring TM, Rueda FC, Tukamoto G, Gasparetto EL. In vivo assessment of white matter damage in neuromyelitis optica: a diffusion tensor and diffusion kurtosis MR imaging study. J Neurol Sci. 2014;345:172-5.

100. Jeantroux J, Kremer S, Lin XZ, Collongues N, Chanson J-B, Bourre B, et al. Diffusion tensor imaging of normal-appearing white matter in neuromyelitis optica. J Neuroradiol. 2012;39: 295-300.

101. Lin F, Yu C, Jiang T, Li K, Zhu C, Zhu W, et al. Discriminative analysis of relapsing neuromyelitis optica and relapsing-remitting multiple sclerosis based on two-dimensional histogram from diffusion tensor imaging. NeuroImage. 2006;31:543-9.

102. Finke C, Heine J, Pache F, Lacheta A, Borisow N, Kuchling J, et al. Normal volumes and microstructural integrity of deep gray matter structures in AQP4+ NMOSD. Neurol Neuroimmunol Neuroinflamm. 2016;3:e229.

103. Liu Y, Duan Y, He Y, Wang J, Xia M, Yu C, et al. Altered topological organization of white matter structural networks in patients with neuromyelitis optica. PLoS One. 2012;7:e48846.

104. Eshaghi A, Riyahi-Alam S, Saeedi R, Roostaei T, Nazeri A, Aghsaei A, et al. Classification algorithms with multi-modal data fusion could accurately distinguish neuromyelitis optica from multiple sclerosis. NeuroImage Clin. 2015;7:306-14.

105. Yu CS, Zhu CZ, Li KC, Xuan Y, Qin W, Sun H, et al. Relapsing neuromyelitis optica and relapsing-remitting multiple sclerosis: differentiation at diffusion-tensor MR imaging of corpus callosum. Radiology. 2007;244:249-56.

106. Kolbe S, Bajraszewski C, Chapman C, Nguyen T, Mitchell P, Paine $\mathrm{M}$, et al. Diffusion tensor imaging of the optic radiations after optic neuritis. Hum Brain Mapp. 2012;33:2047-61.
107. Frohman EM, Dwyer MG, Frohman T, Cox JL, Salter A, Greenberg BM, et al. Relationship of optic nerve and brain conventional and non-conventional MRI measures and retinal nerve fiber layer thickness, as assessed by OCT and GDx: a pilot study. J Neurol Sci. 2009;282:96-105.

108. Naismith RT, Xu J, Tutlam NT, Trinkaus K, Cross AH, Song S-K. Radial diffusivity in remote optic neuritis discriminates visual outcomes. Neurology. 2010;74:1702-10.

109. Smith SA, Williams ZR, Ratchford JN, Newsome SD, Farrell SK, Farrell JAD, et al. Diffusion tensor imaging of the optic nerve in multiple sclerosis: association with retinal damage and visual disability. Am J Neuroradiol. 2011;32:1662-8.

110. Naismith RT, Xu J, Tutlam NT, Snyder A, Benzinger T, Shimony $\mathrm{J}$, et al. Disability in optic neuritis correlates with diffusion tensorderived directional diffusivities. Neurology. 2009;72:589-94.

111. van der Walt A, Kolbe SC, Wang YE, Klistorner A, Shuey N, Ahmadi G, et al. Optic nerve diffusion tensor imaging after acute optic neuritis predicts axonal and visual outcomes. PLoS One. 2013;8:e83825.

112. Naismith RT, Xu J, Tutlam NT, Lancia S, Trinkaus K, Song SK, et al. Diffusion tensor imaging in acute optic neuropathies: predictor of clinical outcomes. Arch Neurol. 2012;69:65-71.

113. Kolbe S, Chapman C, Nguyen T, Bajraszewski C, Johnston L, Kean M, et al. Optic nerve diffusion changes and atrophy jointly predict visual dysfunction after optic neuritis. NeuroImage. 2009;45:679-86.

114. Kolbe SC, Marriott M, van der Walt A, Fielding J, Klistorner A, Mitchell PJ, et al. Diffusion tensor imaging correlates of visual impairment in multiple sclerosis and chronic optic neuritis. Invest Opthalmol Vis Sci. 2012;53:825-32.

115. Rocca MA, Mesaros S, Preziosa P, Pagani E, Stosic-Opincal T, Dujmovic-Basuroski I, et al. Wallerian and trans-synaptic degeneration contribute to optic radiation damage in multiple sclerosis: a diffusion tensor MRI study. Mult Scler. 2013;19:1610-7.

116. Manogaran P, Vavasour IM, Lange AP, Zhao Y, McMullen K, Rauscher A, et al. Quantifying visual pathway axonal and myelin loss in multiple sclerosis and neuromyelitis optica. NeuroImage Clin. 2016;11:743-50.

117. Manogaran P, Hanson JVM, Olbert ED, Egger C, Wicki C, GerthKahlert C, et al. Optical coherence tomography and magnetic resonance imaging in multiple sclerosis and neuromyelitis optica spectrum disorder. Int J Mol Sci. 2016;17:E1894.

118. Harrison DM, Shiee N, Bazin P-L, Newsome SD, Ratchford JN, Pham D, et al. Tract-specific quantitative MRI better correlates with disability than conventional MRI in multiple sclerosis. J Neurol. 2013;260:397-406.

119. Veauthier C, Hasselmann H, Gold SM, Paul F. The Berlin treatment algorithm: recommendations for tailored innovative therapeutic strategies for multiple sclerosis-related fatigue. EPMA J. 2016;7:25.

120. Hagan S, Martin E, Enríquez-de-Salamanca A. Tear fluid biomarkers in ocular and systemic disease: potential use for predictive, preventive and personalised medicine. EPMA J. 2016;7:15.

121. Konieczka K, Koch S, Binggeli T, Schoetzau A, Kesselring J. Multiple sclerosis and primary vascular dysregulation (Flammer syndrome). EPMA J. 2016;7:13.

122. Tournier J-D, Yeh C-H, Calamante F, Cho K-H, Connelly A, Lin C-P. Resolving crossing fibres using constrained spherical deconvolution: validation using diffusion-weighted imaging phantom data. NeuroImage. 2008;42:617-25. 